\title{
Short-term clinical outcomes of Kyocera Modular Limb Salvage System designed cementless stems for the endoprosthetic reconstruction of lower extremities: A Japanese Musculoskeletal Oncology Group multiinstitutional Study
}

Satoshi Tsukushi ( $\nabla$ s-tsuku@aichi-cc.jp)

Aichi Cancer Center

Yoshihiro Nishida

Nagoya University Hospital

Takeshi Hirose

National Cancer Center Hospital

Eiji Nakata

Okayama University Graduate School of Medicine

Rumi Nakagawa

Keio University School of Medicine

Jungo Imanishi

Saitama Medical University International Medical Center

Tomoki Nakamura

Mie University Graduate School of Medicine

Akihito Nagano

Gifu University School of Medicine

Hironari Tamiya

Osaka International Cancer Institute

Takafumi Ueda

National Hospital Organization Osaka National Hospital

Japanese Musculoskeletal Oncology Group

Japanese Musculoskeletal Oncology Group

Research Article

Keywords: endoprosthesis, cementless stem, complication, stress shielding

Posted Date: February 18th, 2022 
DOI: https://doi.org/10.21203/rs.3.rs-1335334/v1

License: (c) (1) This work is licensed under a Creative Commons Attribution 4.0 International License. Read Full License 


\section{Abstract \\ Background}

The high rate of aseptic loosening of cemented stems has led to their frequent use in endoprosthetic reconstruction. However, problems, such as stem breakage and stress shielding at the insertion site, remain. The Japanese Musculoskeletal Oncology Group (JMOG) has developed Kyocera Modular Limb Salvage System (KMLS) cementless stems with a unique tapered press-fit and short fixation design. This study aimed to clarify the short-term postoperative outcomes of this prosthesis and validate the stem design.

\section{Methods}

One hundred cases of KMLS cementless stems (51 male patients; median age, 49 years; mean follow-up period, 35 months), with a minimum follow-up of 2 years, for the proximal femur (PF), distal femur (DF), and proximal tibia were prospectively registered for use. Prosthesis survival, complication rates, postoperative functional, and radiographical evaluation were analyzed. Complications or failures after insertion of the KMLS endoprostheses were classified into five types and functional results were analyzed according to the MSTS scoring system at postoperative 1 year. The diaphyseal interface and anchorage were graded by the ISOLS system at postoperative 2 years.

\section{Results}

The overall prosthesis survival rates at 2 and 4 years were $88.2 \%$ and $79.6 \%$, respectively. The prosthesisspecific survival rate excluding infection and tumor recurrence was $90.2 \%$ and $87.9 \%$, respectively. Younger age $(p=0.045)$ and primary tumor $(p=0.057)$ were associated with poor prognosis of prosthesis-specific survival excluding infection and tumor recurrence. Complications were observed in 31 patients, 13 patients underwent revision surgery. The mean MSTS functional score at 1 year postoperatively was $68 \%$. Early implant loosening was significantly more common in the DF $(p=0.006)$ and PF/DF straight stem $(p=0.038)$. The ISOLS radiographic evaluation at 2 years after surgery revealed good bone remodeling and anchorage in most cases (bone remodeling: 90\% / excellent and good, anchorage: $97 \%$ / excellent and good).

\section{Conclusions}

Tumor endoprosthesis long-term fixation to the diaphysis of the lower extremity remains challenging. The KMLS cementless stem with a unique tapered press fit design showed good short-term results in maintaining bone stock. To prevent early loosening, a curved stem should be used in PF and DF but longterm follow-up is necessary. 


\section{Background}

Due to advances in multidisciplinary approaches for malignant bone tumors, such as Ewing's sarcoma and osteosarcoma, and the development of various endoprostheses, limb-salvage surgery has become the gold standard [1,2]. The prognosis for patients with musculoskeletal sarcomas has improved significantly over the past three decades. Consequently, the durability and longevity of these endoprosthetic implants are of great importance [3-6].

The long-term fixation of the tumor endoprosthesis to the diaphysis of long bones is very challenging, and the optimal method remains controversial. These endoprostheses endure very high rotational stresses at the bone-prosthesis interface due to various factors (young age, high levels of physical activity, loss of long segments, loss of static ligamentous stabilizers, and extensive muscle resection). Historically, cemented prostheses were used due to their immediate stability, which allows for early weight-bearing [7-13]. However, further studies have shown high rates of aseptic loosening in patients with long-term follow-up $[4,10,13,14]$. The reported prosthetic survivorship was $88 \%$ at 2 years, $82 \%$ at 5 years, and $59 \%$ at 10 years [4]. Therefore, orthopedic oncologists frequently use cementless stems to improve long-term implant survival $[15,16,17]$.

The early cementless stems incorporated a side plate and screw components. As it is associated with very high rates of intramedullary fixation, aseptic loosening is rare but various failures such as stem breakage, screw loosening, and stress shielding are often experienced [16-19]. Various forms of press-fit cementless stem with or without flutes have been developed [20-22]. Most press-fit cementless stems have long extensively porous-coated intramedullary stems allowing bony growth over the whole length of the stem. However, intramedullary fixation is inherently unphysiological and results in reduced loading of the surrounding cortical bone. This results in stress shielding of the surrounding bone and an increased risk of aseptic loosening. However, the optimal design to reduce stress shielding is controversial.

Tumor endoprostheses that are used worldwide are generally designed for Caucasian body types and are frequently too large or too heavy for Asian-pacific patients. The Japanese Musculoskeletal Oncology Group (JMOG) has been involved in modifying small and light modular prothesis (Physio Hinge Total Knee System Type III/PHK III, Kyocera Modular Limb Salvage System [KMLS]) that are suitable for Asianpacific patients $[19,23]$. KMLS has developed a newly designed cementless stem with a unique tapered press-fit and short fixation design for the proximal femur (PF), distal femur (DF), and proximal tibia (PT) that has been in use since August 2014. From August 2014 to March 2018, 100 cases of KMLS newly designed cementless stems were prospectively registered for use at JMOG-affiliated institutions.

The purpose of this study was to clarify the short-term postoperative results of the cementless stem with a unique tapered press-fit design, confirm the validity of the stem design, and provide an index for future improvement.

\section{Methods}




\section{Study Design and Setting}

From August 2014 to March 2018, 100 cases of KMLS newly designed cementless stems for the PF, DF, and PT were prospectively registered for use at JMOG-affiliated 14 institutions. Following institutional review board approval, we retrospectively reviewed clinical outcomes of 100 newly designed cementless stems with a minimum follow-up of 2 years in March 2020.

The records of all patients were collected using a questionnaire administered to the members of the JMOG. The collected data included the demographic details, histological diagnosis, surgical stage, adjuvant therapy, size of the prosthetic components, complications, 1-year postoperative MSTS functional score, 2-year ISOLS radiographic evaluation, and oncological outcome at the final follow-up.

The primary endpoint of this study was to identify the prosthesis survival and the implant complication/failure rates. The secondary endpoint was to determine the radiological and functional outcomes.

\section{Demographics, Description of Study Population}

A total of 100 cases of KMLS newly designed cementless stems for the PF, DF, and PT fit the inclusion criteria (Table 1). There were 51 males and 49 females. The median age was 49 years. Follow-up was at a minimum of 2 years (mean, 35 months; range, 24-53 months). Four (4\%) patients were lost to followup. Anatomical locations were as follows: PF $(n=49), D F(n=39)$, and PT $(n=12)$. There were 64 primary tumors ( 57 bone tumors and 7 soft tissue sarcomas) and 36 metastatic bone tumors. The primary bone tumors included 35 osteosarcomas, eight chondrosarcomas, eight giant cell tumors of bone, five undifferentiated pleomorphic sarcomas, and one leiomyosarcoma. The primary soft tissue sarcomas included two undifferentiated pleomorphic sarcomas, two leiomyosarcoma, two synovial sarcomas, and one liposarcoma. Chemotherapy was administered in 57 patients and irradiation in three patients. Extraarticular resections were performed in seven patients of DF and a patient of PF. For the cementless stems of the PF and DF, straight and curved stems can be used selectively. In the PF, a straight stem was used in eight (16\%) of 49 cases. In the DF, a straight stem was used in eight (21\%) of 39 cases.

\section{Prosthesis design}

The KMLS system is an original prosthesis of JMOG and is designed especially for patients with an Asian body type (Fig. 1). The metallic parts of the KMLS are made of lightweight and high-strength titanium alloy (Ti-6Al-4V) with good biocompatibility and bio-stability. The base of the stem has a tapered press-fit design, and the interface is processed by porous proofing made of pure titanium to promote bone ingrowth. The cementless stems of the PF and DF have derotational flutes to provide adequate initial rotational stability, and straight and curved stems can be used selectively. This prosthesis has not been approved for that use by the United States Food and Drug Administration.

\section{Variables, Outcome Measures, and Data Sources}


We calculated the relative prosthetic-shaft diameter by dividing the diaphysis diameter by prosthetic diameter (diaphysis/stem coefficient) at the midpoint of the prosthetic stem (Fig. 2). It has been reported that the diaphyseal/stem coefficient of cementless stem over 2.5 predicted lower prosthetic survival [24].

The diaphyseal interface and anchorage were graded by the ISOLS system [25] at postoperative 2 years; 65 of the 100 were available for ISOLS radiological evaluation (65\%).

Functional results were analyzed according to the 30-point functional Musculoskeletal Tumor Society (MSTS) scoring system [26] at 1 year postoperatively; 72 of the 100 were available for 1-year postoperative MSTS functional score (72\%).

Complications or failures after insertion of the KMLS endoprostheses were classified according to Henderson et al. [27] in five different types: type 1 (mechanical failure due to soft tissue problems, such as debridement, peroneal nerve palsy, dislocation of joint [closed reduction]) and superficial infections), type 2 (aseptic loosening), type 3 (structural failures, such as periprosthetic fractures and hip dislocation requiring surgical treatment), type 4 (non-mechanical failures, such as deep infection), and type 5 (tumor progression).

\section{Statistical analysis}

The statistical associations between the clinicopathological factors and complications were evaluated using the Fisher's exact test or Chi-square test. The duration of survival was defined as the interval between the date of initial treatment for the primary tumor and the date of death. Patients who died from non-tumor-related causes were uncensored at the time of death in this study. The overall prosthesis survival rate was defined as the time from surgical reconstruction using the KMLS system to revision surgery due to any prosthetic failure including minor parts of the prosthesis, due to local recurrence, polyethylene bushing failure, breakage of the prosthesis, aseptic loosening, or infection. The prosthesisspecific survival rate was defined as the time from surgical reconstruction using the KMLS system to revision surgery due to implant failure excluding infection and tumor recurrence. The limb salvage rate was calculated as the time from surgical reconstruction using the KMLS system to amputation. Survival curves were constructed using the Kaplan-Meier method. The subgroups were compared using the logrank test. The level of statistical significance was set at $p<0.05$ and confidence intervals were reported at $95 \%(95 \% \mathrm{Cl})$.

\section{Oncologic Results}

At the final follow-up, 59 patients were alive, and 41 patients had died of disease. The overall survival rates at 2 and 4 years were $88.2 \%$ and $79.6 \%$, respectively. Twelve patients developed local recurrence. The limb salvage rate at 2 and 4 years was $98.9 \%$ and $96.9 \%$, respectively. Two patients (osteosarcoma and undifferentiated pleomorphic sarcoma of bone) underwent amputations for local recurrences.

\section{Results}




\section{Prosthesis survival and prosthesis-specific survival}

The overall prosthesis survival rates at 2 and 4 years were $88.2 \%$ and $79.6 \%$, respectively (Fig. $3 \mathrm{~A}$ ). Male gender $(p=0.063)$ and primary tumor $(p=0.08)$ were associated with poor prognosis of overall prosthesis survival (Table 2). The prosthesis-specific survival rate excluding infection and tumor recurrence at 2 and 4 years were $90.2 \%$ and $87.9 \%$, respectively (Fig. 3B). Younger age $(p=0.045)$ and primary tumor $(p=0.057)$ were associated with poor prognosis of prosthesis-specific survival excluding infection and tumor recurrence (Table 3) (Fig. 4A-C).

\section{Radiographic and functional evaluation}

The ISOLS radiographic evaluation at 2 years after surgery revealed good bone remodeling and anchorage in most cases ( $72 \%$ excellent, $18 \%$ good, $8 \%$ fair, and $2 \%$ poor bone remodeling around the implant and $92 \%$ excellent, $5 \%$ good, and $2 \%$ fair for the anchorage itself). The mean MSTS functional score at 1 year postoperatively was $68 \%$.

\section{Complication}

In total, 31 failures/complications (31\%) were observed in $100 \mathrm{KMLS}$ newly designed cementless stems, and the anatomical location and failure classifications according to Henderson et al. are summarized in Table 4.

No type 1 failure (soft tissue related) occurred. Type 2 failure (aseptic loosening) occurred in 10 patients (PF/DF/PT = 1/8/1). All cases were caused by loosening of the component due to rotational instability at an early phase (nine new cementless stems and one femoral component of PT). Six of the 10 patients underwent revision surgery, and four were treated conservatively. Type 3 failure (structural) occurred in three patients (PF/DF/PT = 2/1/0) and included hip dislocation $(n=1)$ and periprosthetic fracture $(n=2)$. All failures were treated surgically. No stem breakage was observed at the final follow-up. Type 4 failure (infections) occurred in six patients (PF/DF/PT = 1/4/1) and included early infection $(n=1)$ and late infection $(n=5)$. All failures were treated surgically and three of six patients underwent revision surgery. Type 5 failure (local tumor progression) occurred in 12 patients (PF/DF/PT= 5/6/1) and included skeletal recurrence $(n=5)$ and soft tissue recurrence $(n=7)$. Two patients (one with osteosarcoma and one undifferentiated pleomorphic sarcoma of bone) underwent amputations for local recurrences. Revision surgery was performed in 13 patients and amputation in two patients. Early implant loosening was significantly more common in the DF $(p=0.006)$ and straight stem of PF/DF $(p=0.038)$.

\section{Discussion}

Ideally, reconstruction of bony defects should be biological. In large defects in curative limb salvage surgery, complete biological reconstruction is limited and, in most cases, large endoprostheses have been used. Long-term durability is required for the fixation of tumor endoprostheses to the diaphysis of long bones. However, the optimal method remains controversial. Previous reports have revealed that cemented 
prostheses have a higher incidence of aseptic loosening $[4,10,13,14]$, which has encouraged many orthopedic oncologists to use cementless stems $[15,16]$. Early cementless stems incorporated side plate and screw components, which often resulted in various failures such as stem breakage, screw loosening, and stress shielding $[15,18,19]$, as such, the development of a new cementless stem is required. KMLS has also developed a newly designed cementless stem with a unique tapered press-fit design for the PF, DF, and PT and has been in use since August 2014. From August 2014 to March 2018, 100 cases of KMLS newly designed cementless stems were prospectively registered for use at JMOG-affiliated institutions. We retrospectively reviewed short-term clinical outcomes of these cementless stems in March 2020 with a minimum follow-up of 2 years. The overall prosthesis survival rates at 2 and 4 years were $88.2 \%$ and $79.6 \%$, respectively. The prosthesis-specific survival rate excluding infection and tumor recurrence at 2 and 4 years were $90.2 \%$ and $87.9 \%$, respectively. The limb salvage rate at 2 and 4 years was $98.9 \%$ and $96.9 \%$, respectively. The KMLS newly designed cementless stem showed good short-term results with a mean follow-up period of 35 months. Previous studies demonstrated that prosthetic survivals with cementless stem fixation ranged from $65.4-96 \%[21,22,28-30]$ but the heterogeneity of the patient population makes it difficult to directly compare the survival results of the present series with those of these previous studies. However, our results are comparable to those previously reported in the literature.

The design of this cementless stem has two features including the tapered press-fit design and the short fixation of the tapered portion. The previous cementless stems incorporated side plate and screw components. Various failures, such as stem breakage, screw loosening, and stress shielding, are often experienced and the tapered design to avoid stem breakage improved the strength of the stem base, where the most stress is concentrated, and there was no stem breakage in this cohort with a mean followup period of 35 months. However, Capanna et al. reported that six of 95 cases of femoral stem breakage occurred at an average of 43 months [17] thus, careful follow-up in the long term is needed. In addition, the tapered press-fit design allows early weight-bearing, which is advantageous for bone ingrowth, and makes the patient ambulatory at an early stage, which is a greatly beneficial to the patient.

Cementless stems with long extensively porous-coated intramedullary stems provide strong anchorage over the whole length of the stem. However, intramedullary long fixation is not physiological and reduces the load on the surrounding cortical bone. This causes stress shielding of the surrounding bone, which leads to aseptic loosening. The short fixation design of this new cementless stem has been improved with a concept that preserves physiological bone loading and bone stock. Notably, the ISOLS radiographic evaluation at 2 years after surgery revealed very good bone remodeling and anchorage in most cases (bone remodeling: 90\% / excellent and good, anchorage: 97\% / excellent and good). Once initial rotational stability was achieved, there was little stress shielding, and the bone stock was preserved (Fig. 5A, B). In our experience, stress shielding often reaches a plateau at 2 years after surgery and stabilizes thereafter, but medium- to long-term follow-up is necessary.

Conversely, in total, 31 failures/complications (31\%) were observed in $100 \mathrm{KMLS}$ newly designed cementless stems. The prosthesis-specific survival rate excluding infection and tumor recurrence at 2 and 
4 years were $90.2 \%$ and $87.9 \%$, respectively. Younger age $(p=0.045)$ and primary tumor $(p=0.057)$ were associated with poor prognosis of prosthesis-specific survival excluding infection and tumor recurrence. Early loosening in nine cases was associated with the new cementless stem. This failure was significantly more common in the DF $(p=0.006)$ and PF/DF straight stem $(p=0.038)$. It is important to obtain the initial rotational stability in the anchorage of the cementless stem in this design. The DF is anatomically prone to rotational stress and is a common site for primary malignant bone tumors such as osteosarcoma, which is associated with younger age and high activity. Therefore, a short fixation with a tapered press-fit is not sufficient to provide reliable rotational stability for high-activity patients, and the use of a curved stem is recommended. Furthermore, the tapered press-fit for thin diaphysis of young patients is often difficult to broach the medullary canal without cracking. Cracking of the host bone might compromise bone healing especially under additional intensive chemotherapy. Some failures with rotational instability occurred in the early stages of this study, but the reaming technique and the development of optional instruments allowing gradual deepening of the grooves in the cortical bone solved these problems over time.

This study has some limitations. First, this was a retrospective review and is subject to those inherent limitations and biases. However, KMLS newly designed cementless stems were prospectively registered at JMOG-affiliated institutions, and data on postoperative function and postoperative radiographic evaluation were collected. Endoprosthesis is a rare procedure and experience at a single institution is limited and it is difficult to perform a prospective evaluation with sufficient statistical power. We believe that it is significant to share the experience of newly designed endoprosthesis at an early stage in a multicenter setting for future development. Second, the duration of patient follow-up is short. In our series, the mean duration of patient follow-up was 35 months (range: 24-53 months). A careful follow-up in the long term will be necessary in the future. However, most failures at the bone-endoprosthesis junction of cementless stem that require revision surgery would be likely to occur very early in the postoperative period. Therefore, we set the registration period to 4 years and the minimum follow-up period to 2 years. Finally, anatomical locations (PF, DF, and PT) are strongly related to diagnosis (primary or metastasis) and age, and interpretation of prosthesis survival and complications should take these biases into account.

\section{Conclusion}

The long-term fixation of tumor endoprosthesis to the diaphysis of the lower extremity remains challenging. JMOG has been involved in improving small and light modular prostheses that are suitable for Asian-pacific patients. KMLS have developed a newly designed cementless stem with a unique tapered press-fit and short fixation design for the PF, DF, and PT. This cementless stem showed good short-term results in preserving bone stock. The short fixation design of this cementless stem has been improved with a concept that preserves physiological bone loading and bone stock. The ISOLS radiographic evaluation at 2 years after surgery revealed very good bone remodeling and anchorage. On the other hand, we experienced several cases of early loosening. To prevent early loosening, straight stem should not be used in PF and DF and broaching in the medullary canal requires great care to avoid 
cracking the bone. In the future, careful long-term follow-up is necessary, and we should consider refining our cementless stem design to account for higher activity and anatomical locations where stress is concentrated, based on the results of this study.

\section{Abbreviations}

$\begin{array}{ll}\text { DF } & \text { Distal femur } \\ \text { JMOG } & \text { Japanese Musculoskeletal Oncology Group } \\ \text { KMLS } & \text { Kyocera Modular Limb Salvage System } \\ \text { PF } & \text { Proximal femur } \\ \text { PT } & \text { Proximal tibia }\end{array}$

\section{Declarations}

\section{Ethics approval and consent to participate}

Written informed consent was obtained from all patients. This study was performed according to the "Declaration of Helsinki" and was approved by the Nagoya University Ethical Research Committee.

\section{Consent for publication}

Written informed consent for publication was obtained from all participants.

The patients shown in Fig. 5A and 5B signed consent for their personal and clinical details along with identifying images to be published in this study.

\section{Availability of data and materials}

All data can be available at request by email to the corresponding author.

\section{Competing interests}

The institutions of two of the authors (YN and JG) has received funding from Kyocera.

Other authors certify that neither he, nor any member of his immediate family, has funding or commercial associations (consultancies, stock ownership, equity interest, patent/licensing arrangements, etc) that might pose a conflict of interest in connection with the submitted article.

\section{Funding}


This research did not receive any specific grant from funding agencies in the public, commercial, or notfor-profit sectors.

\section{Availability of data and materials}

All data can be available at request by email to the corresponding author.

\section{Authors' contributions}

ST, TH, EN, RN, JI, TN, AN, HT and JG collected clinical data, participated in the data interpretation, and drafted the manuscript. ST and YN collected and analyzed the data. TH, EN, RN, JI, TN, AN and HT participated in statistics and manuscript revision. ST and YN participated in the design of the study and approved the revised manuscript. ST, YN and TU conceived idea for the study, participated in its design and coordination, and helped to draft the manuscript. All authors read and approved the final manuscript.

\section{Acknowledgements}

We thank the following members of the JMOG for their cooperation with collection of clinical data of patients: Kakunaga, K Honoki, R Terauchi, H Outani, S Nishimura, I Kunihiro.

We would like to thank Editage (www.editage.com) for English language editing.

\section{References}

1. Hwang JS, Mehta AD, Yoon RS, Beebe KS. From amputation to limb salvage reconstruction: evolution and role of the endoprosthesis in musculoskeletal oncology. J Orthop Traumatol. 2014;15:81-6.

2. Aksnes LH, Bauer HC, Jebsen NL, Follerås G, Allert C, Haugen GS, et al. Limb-sparing surgery preserves more function than amputation: a Scandinavian sarcoma group study of 118 patients. $\mathrm{J}$ Bone Joint Surg Br. 2008;90:786-94.

3. Eckardt JJ, Safran MR, Eilber FR, Rosen G, Kabo JM. Expandable endoprosthetic reconstruction of the skeletally immature after malignant bone tumor resection. Clin Orthop Relat Res. 1993;297:188202.

4. Morgan HD, Cizik AM, Leopold SS, Hawkins DS, Conrad EU 3rd. Survival of tumor megaprostheses replacements about the knee. Clin Orthop Relat Res. 2006;450:39-45.

5. Ferrari S, Palmerini E. Adjuvant and neoadjuvant combination chemotherapy for osteogenic sarcoma. Curr Opin Oncol. 2007;19:341-6.

6. Mirabello L, Troisi RJ, Savage SA. Osteosarcoma incidence and survival rates from 1973 to 2004: data from the surveillance, epidemiology, and end results program. Cancer. 2009;115:1531-43.

7. Lempberg R, Ahlgren O. Prosthetic replacement of tumour-destroyed diaphyseal bone in the lower extremity. Acta Orthop Scand. 1982;53:541-5. 
8. Horowitz SM, Glasser DB, Lane JM, Healey JH. Prosthetic and extremity survivorship after limb salvage for sarcoma. How long do the reconstructions last? Clin Orthop Relat Res. 1993;:280-6.

9. Schindler OS, Cannon SR, Briggs TW, Blunn GW, Grimer RJ. Walker PS. Use of extendable total femoral replacements in children with malignant bone tumors. Clin Orthop Relat Res. 1998;357:15770.

10. Kawai A, Lin PP, Boland PJ, Athanasian EA, Healey JH. Relationship between magnitude of resection, complication, and prosthetic survival after prosthetic knee reconstructions for distal femoral tumors. J Surg Oncol. 1999;70:109-15.

11. Shin DS, Choong PF, Chao EY, Sim FH. Large tumor endoprostheses and extracortical bone-bridging: 28 patients followed 10-20 years. Acta Orthop Scand. 2000;71:305-11.

12. Sharma S, Turcotte RE, Isler MH, Wong C. Experience with cemented large segment endoprostheses for tumors. Clin Orthop Relat Res. 2007;459:54-9.

13. Wirganowicz PZ, Eckardt JJ, Dorey FJ, Eilber FR, Kabo JM. Etiology and results of tumor endoprosthesis revision surgery in 64 patients. Clin Orthop Relat Res. 1999;358:64-74.

14. Unwin P Unwin PS, Cannon SR, Grimer RJ, Kemp HB, Sneath RS, Walker PS. Aseptic loosening in cemented custom-made prosthetic replacements for bone tumours of the lower limb. J Bone Joint Surg Br. 1996;78:5-13.

15. Griffin AM, Parsons JA, Davis AM, Bell RS, Wunder JS. Uncemented tumor endoprostheses at the knee: root causes of failure. Clin Orthop Relat Res. 2005;438:71-9.

16. Flint MN, Griffin AM, Bell RS, Ferguson PC, Wunder JS. Aseptic loosening is uncommon with uncemented proximal tibia tumor prostheses. Clin Orthop Relat Res. 2006;450:52-9.

17. Capanna R, Morris HG, Campanacci D, Del Ben M, Campanacci M. Modular uncemented prosthetic reconstruction after resection of tumours of the distal femur. J Bone Joint Surg Br. 1994;76:178-86.

18. Hauer TM, Houdek MT, Bhumbra R, al. Component fracture in the Kotz modular femoral tibial reconstruction system:an under-reported complication. J Arthroplasty. 2018;33:544-7.

19. Nakamura T, Matsumine A, Uchida A, Kawai A, Nishida Y, Kunisada T, et al. Clinical outcomes of Kyocera Modular Limb Salvage system after resection of bone sarcoma of the distal part of the femur: the Japanese Musculoskeletal Oncology Group study. Int Orthop. 2014;38:825-30.

20. Ferguson PC, Zdero R, Schemitsch EH, Deheshi BM, Bell RS, Wunder JS. A biomechanical evaluation of press-fit stem constructs for tumor endoprosthetic reconstruction of the distal femur. $J$ Arthroplasty. 2011;26:1373-9.

21. Visgauss JD, Perrin DL, Wilson DA, al. Midterm success of a custom, non-fluted, diaphyseal, press-fit stem used with a tumor megaprosthesis system. J Arthroplasty. 2020;35:1333-8.

22. Hu CC, Chen SY, Chen CC, Chang YH, Ueng SW, Shih HN. Superior survivorship of cementless vs cemented diaphyseal fixed modular rotating-hinged knee megaprosthesis at 7 years' follow-Up. J Arthroplasty. 2017;32:1940-5. 
23. Matsumine A, Ueda T, Sugita T, Yazawa Y, Isu K, Kawai A, et al. Clinical outcomes of the Kyocera Physio Hinge Total Knee System Type III after the resection of a bone and soft tissue tumor of the distal part of the femur. Japanese Musculoskeletal Oncology Group. J Surg Oncol. 2011;103:25763.

24. Farfalli GL, Boland PJ, Morris CD, Athanasian EA, Healey JH. Early equivalence of uncemented pressfit and compress femoral fixation. Clin Orthop Relat Res. 2009;467:2792-9.

25. Glasser D, Langlais $F$. The ISOLS radiological implant evaluation system. In: Langlais F, Tomeno B, editors. Limb Salvage: Major Reconstructions in Oncologic and Nontumoral Conditions. Springer, Heidelberg, Berlin, New York, Tokyo;1991.

26. Enneking WF, Dunham W, Gebhardt MC, Malawar M, Pritchard DJ. A system for the functional evaluation of reconstructive procedures after surgical treatment of tumors of the musculoskeletal system. Clin Orthop Relat Res. 1993;286:241-6.

27. Henderson ER, Groundland JS, Pala E, Dennis JA, Wooten R, Cheong D, et al. Failure mode classification for tumor endoprostheses: retrospective review of five institutions and a literature review. J Bone Joint Surg Am. 2011;93:418-29.

28. Wirganowicz PZ, Eckardt JJ, Dorey FJ, Eilber FR, Kabo JM. Etiology and results of tumor endoprosthesis revision surgery in 64 patients. Clin Orthop Relat Res. 1999;358:64-74.

29. Pala E, Mavrogenis AF, Angelini A, Henderson ER, Douglas Letson G, Ruggieri P. Cemented versus cementless endoprostheses for lower limb salvage surgery. J BUON. 2013;18:496-503.

30. Bekmez S, Ayvaz M, Yucekul A, Tokgozoglu M. Modular cementless prosthetic reconstruction after resection of lower extremity malignant tumor. Acta Orthop Traumatol Turc. 2016;50:674-80.

\section{Tables}

TABLE 1 Clinicopathological data on 100 cases of reconstruction with the newly designed cementless stem 
Characteristic

\begin{tabular}{llcl} 
All & Proximal & Distal & Proximal \\
& femur & femur & tibia \\
\hline$(\mathrm{N}=100)$ & $(\mathrm{N}=49)$ & $(\mathrm{N}=39)$ & $(\mathrm{N}=12)$
\end{tabular}

Age

median

$\geq 50 \mathrm{yr}$

$₫ 50 \mathrm{yr}$

Gender

male

female

Diagnosis

primary

bone tumor

osteosarcoma

chondrosarcoma

giant cell tumor of bone

undifferentiated pleomorphic sarcoma leiomyosarcoma

soft tissue tumor

undifferentiated pleomorphic sarcoma 2

leiomyosarcoma

synovial sarcoma

liposarcoma

metastatic bone tumor

BMI (body mass index)

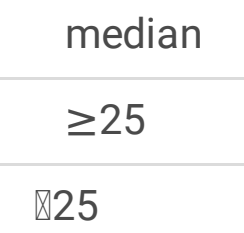

With chemotherapy

\begin{tabular}{llll}
\hline $49.0 \mathrm{yr}$ & $56.0 \mathrm{yr}$ & $30.0 \mathrm{yr}$ & $34.5 \mathrm{yr}$ \\
\hline 48 & 32 & 11 & 5 \\
\hline 52 & 17 & 28 & 7
\end{tabular}

$\begin{array}{llll}51 & 19 & 25 & 7 \\ 49 & 30 & 14 & 5\end{array}$

$\begin{array}{llll}64 & 18 & 35 & 11\end{array}$

$\begin{array}{llll}57 & 13 & 33 & 11\end{array}$

$\begin{array}{llll}35 & 4 & 23 & 8\end{array}$

$\begin{array}{llll}8 & 5 & 3 & 0\end{array}$

$\begin{array}{llll}8 & 1 & 6 & 1\end{array}$

$\begin{array}{llll}5 & 3 & 0 & 2\end{array}$

$\begin{array}{llll}1 & 0 & 1 & 0\end{array}$

$\begin{array}{llll}7 & 5 & 2 & 0\end{array}$

$2 \quad 2$

$2 \quad 2$

$\begin{array}{lll}2 & 1 & 1\end{array}$

1 1

36

31

4

1

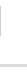

\section{2}

0

0

2

2

1

1

$\begin{array}{llll}20.7 & 20.3 & 21.3 & 20.6 \\ 11 & 5 & 4 & 2 \\ 89 & 44 & 35 & 10 \\ 57 & 26 & 24 & 7\end{array}$




\begin{tabular}{|lllll}
\hline Without chemotherapy & 43 & 23 & 15 & 5 \\
\hline With radiation & 3 & 2 & 1 & 0 \\
\hline Without radiation & 97 & 47 & 38 & 12 \\
\hline diaphyseal/stem coefficient & & & & \\
\hline \multicolumn{1}{c}{ median } & 2.33 & 2.37 & 2.38 & 1.90 \\
\hline 2.50 & 30 & 17 & 12 & 1 \\
\hline 2.50 & 70 & 32 & 27 & 11
\end{tabular}

TABLE 2 The relationship between overall prosthesis survival and patient characteristics

\begin{tabular}{|c|c|c|c|c|}
\hline Variables & & $\mathbf{n}$ & 2-year survival (\%) & $p$ value \\
\hline \multirow[t]{2}{*}{ Age } & $\geq 50 \mathrm{yr}$ & 48 & 94.6 & $p=0.154$ \\
\hline & $\nabla 50 \mathrm{yr}$ & 52 & 83.4 & \\
\hline \multirow[t]{2}{*}{ Gender } & male & 51 & 83.1 & $p=0.063$ \\
\hline & female & 49 & 93.4 & \\
\hline \multirow[t]{2}{*}{ Diagnosis } & primary & 64 & 85.1 & $p=0.080$ \\
\hline & metastasis & 36 & 96.2 & \\
\hline \multirow[t]{3}{*}{ Location } & proximal femur & 49 & 93.6 & $p=0.279$ \\
\hline & distal femur & 39 & 81.5 & \\
\hline & proximal tibia & 12 & 90.9 & \\
\hline \multirow[t]{2}{*}{ BMI } & $\geq 25$ & 11 & 79.5 & $p=0.188$ \\
\hline & 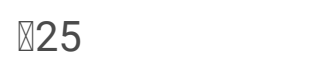 & 89 & 89.3 & \\
\hline \multirow[t]{2}{*}{ Chemotherapy } & yes & 57 & 89.1 & $p=0.783$ \\
\hline & no & 43 & 86.8 & \\
\hline \multirow[t]{2}{*}{ Radiation } & yes & 3 & 100 & $p=0.240$ \\
\hline & no & 97 & 87.8 & \\
\hline \multirow[t]{2}{*}{ Diaphyseal/stem coefficient } & $\geq 2.50$ & 30 & 84.3 & $p=0.203$ \\
\hline & $\nabla 2.50$ & 70 & 89.9 & \\
\hline
\end{tabular}


TABLE 3 The relationship between prosthesis specific-survival and patient characteristics

\begin{tabular}{|c|c|c|c|c|}
\hline Variables & & $\mathbf{n}$ & 2-year survival (\%) & $\mathrm{p}$ value \\
\hline \multirow[t]{2}{*}{ Age } & $\geq 50 \mathrm{yr}$ & 48 & 97.1 & $p=0.045^{\star}$ \\
\hline & $\nabla 50 \mathrm{yr}$ & 52 & 85.2 & \\
\hline \multirow[t]{2}{*}{ Gender } & male & 51 & 85 & $p=0.298$ \\
\hline & female & 49 & 95.5 & \\
\hline \multirow[t]{2}{*}{ Diagnosis } & primary & 64 & 86.5 & $p=0.057$ \\
\hline & metastasis & 36 & 100 & \\
\hline \multirow[t]{3}{*}{ Location } & proximal femur & 49 & 93.6 & $p=0.275$ \\
\hline & distal femur & 39 & 86.3 & \\
\hline & proximal tibia & 12 & 80.9 & \\
\hline \multirow[t]{2}{*}{ BMI } & $\geq 25$ & 11 & 79.5 & $p=0.246$ \\
\hline & $\otimes 25$ & 89 & 96.3 & \\
\hline \multirow[t]{2}{*}{ Chemotherapy } & yes & 57 & 90.9 & $p=0.561$ \\
\hline & no & 43 & 89.3 & \\
\hline \multirow[t]{2}{*}{ Radiation } & yes & 3 & 100 & $p=0.622$ \\
\hline & no & 97 & 90 & \\
\hline \multirow[t]{2}{*}{ Diaphyseal/stem coefficient } & $\geq 2.50$ & 30 & 91.5 & $p=0.630$ \\
\hline & $₫ 2.50$ & 70 & 89.9 & \\
\hline
\end{tabular}

* a statistically significant $p$ value.

TABLE 4 Causes of prosthesis failure 


\begin{tabular}{lllllll}
$\begin{array}{l}\text { Type of } \\
\text { complication }\end{array}$ & Type 1 & Type 2 & Type 3 & Type 4 & Type 5 & All types \\
\hline (n) & $\begin{array}{l}\text { soft } \\
\text { tissue }\end{array}$ & aseptic & structural & infection & tumor & \\
\hline & failure & loosening & failure & & progression & \\
\hline Proximal femur (49) & 0 & 1 & 2 & 1 & 5 & 6 \\
\hline Distal femur (39) & 0 & 8 & 1 & 4 & 1 & 31 \\
\hline Proximal tibia (12) & 0 & 1 & 0 & 1 & 12 &
\end{tabular}

Figures 


$$
19
$$




\section{Figure 2}

Coefficient diaphysis/stem. This coefficient was obtained through the division between the diaphysis diameter and the prosthetic-stem at the midpoint of the prosthetic-stem.

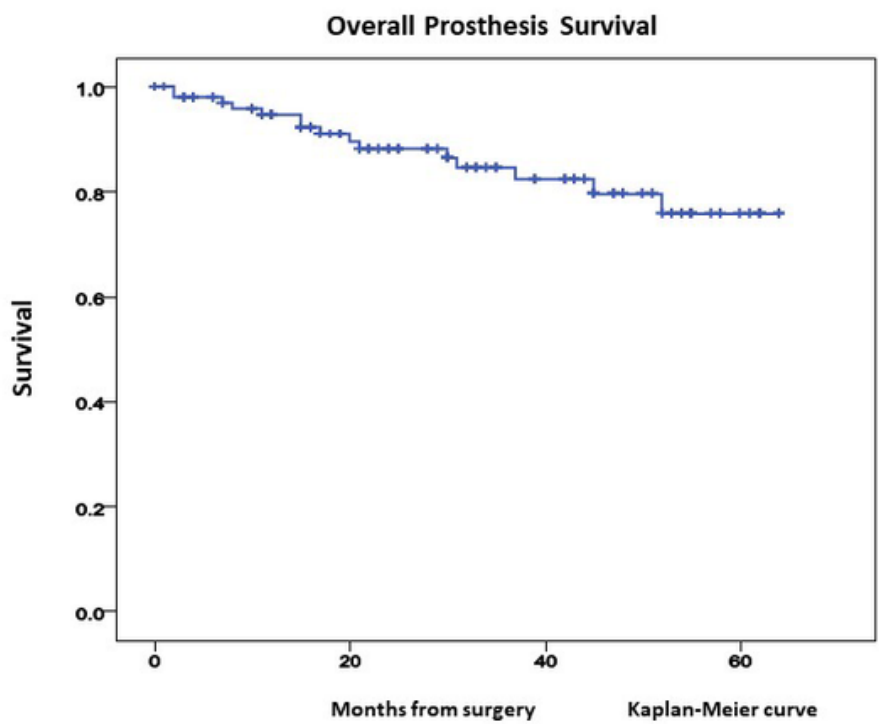

A

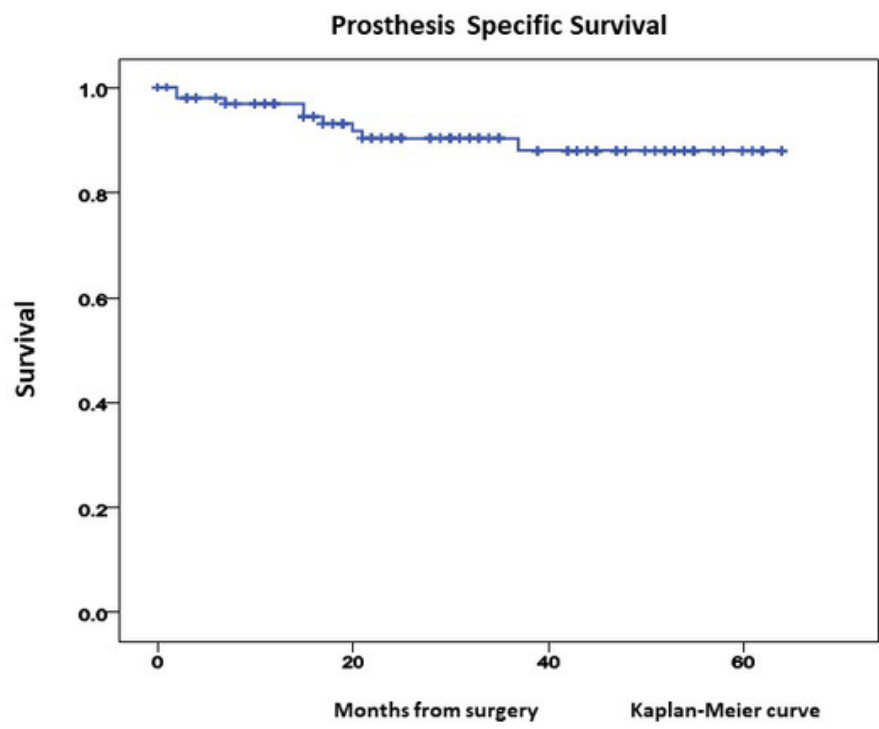


Survivorship of the KMLS newly designed cementless stem. A. Overall survivorship of the KMLS newly designed cementless stem. B. Prosthesis specific survivorship of the KMLS newly designed cementless stem.

KMLS, Kyocera Modular Limb Salvage System
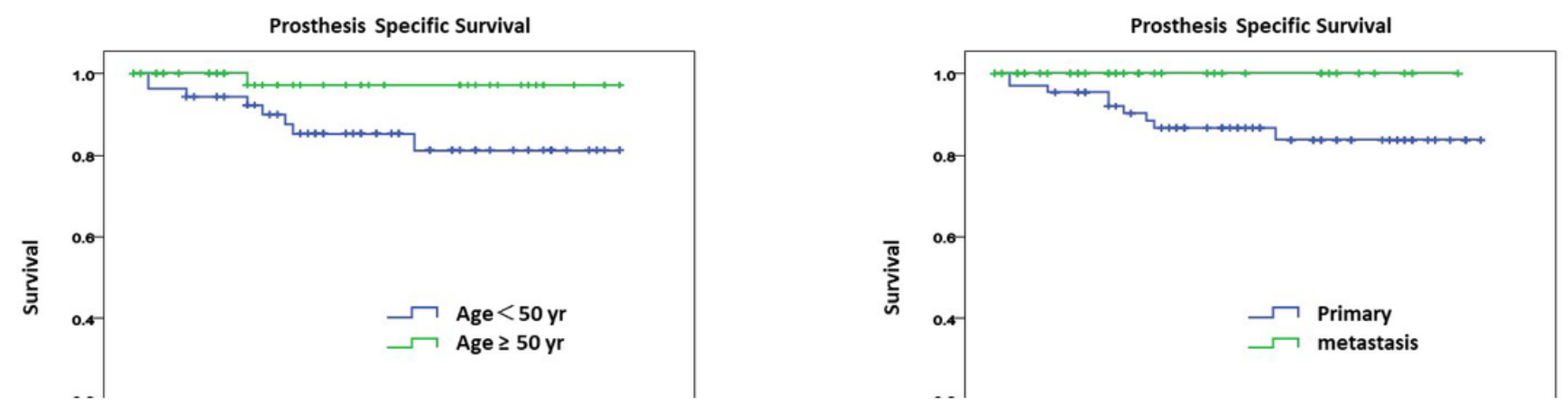

\section{Figure 4}

Kaplan-Meier survival curves showing prosthesis survival of the KMLS newly designed cementless stem in A. younger and older groups, B. primary and metastasis groups and C. in proximal femur, distal femur, and proximal tibia groups.KMLS, Kyocera Modular Limb Salvage System 


\section{Figure 5}

A 3-year postoperative radiograph showing the lateral view of the KMLS newly designed cementless stem in a patient with $\mathrm{A}$. giant bone cell tumor and $\mathrm{B}$. bone metastases from breast cancer.

KMLS, Kyocera Modular Limb Salvage System 